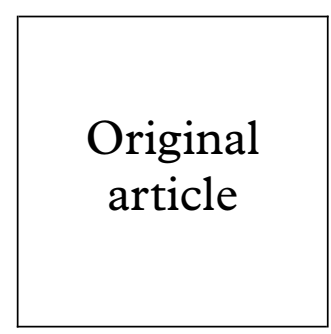

\section{Department of Medicine, Makerere \\ Medical School, \\ Kampala, Uganda \\ E T Katabira \\ N K Sewankambo \\ R D Mugerwa \\ F X Mubiru \\ C Othieno}

Global Programme on AIDS, World Health

Organisation, Geneva,

Switzerland

E M Belsey

M Karam

M Youle

J H Perriens

J M A Lange

Nakasero Blood

Transfusion Centre,

Kampala, Uganda

P Kataaha

Correspondence to:

Dr E T Katabira,

Department of Medicine,

Makerere Medical School,

PO Box 7072, Kampala,

Uganda.

Accepted for publication 5 March 1998

\title{
Lack of efficacy of low dose oral interferon alfa in symptomatic HIV-1 infection: a randomised, double blind, placebo controlled trial
}

\author{
Elly T Katabira, Nelson K Sewankambo, Roy D Mugerwa, Elizabeth M Belsey, \\ Francis X Mubiru, Catherine Othieno, Peter Kataaha, Marc Karam, Mike Youle, \\ Joseph H Perriens, Joep M A Lange
}

Background: Interferon alfa (IFN- $\alpha$ ) exhibits dose related in vitro activity against human immunodeficiency virus (HIV), with complete inhibition of HIV replication at IFN- $\alpha$ concentrations $\geqslant 256 \mathrm{IU} / \mathrm{ml}$. In mid-1990, Kenyan investigators reported that oral administration of an extremely low dose (150 IU/day) of natural human ( $\mathrm{nHu}$ ) IFN- $\alpha$ resulted in complete alleviation of AIDS related complex and AIDS symptoms and resolution of opportunistic infections without additional treatment. Moreover, loss of HIV antibody seropositivity was reported in approximately $10 \%$ of treated patients. Subsequent small studies failed to substantiate these spectacular claims, but controversy on the efficacy of this treatment persisted.

Methods: We studied 559 adult Ugandan patients with WHO stage 2-4 HIV infection and a Karnofsky performance score of more than 50, who had not received any drugs with antiretroviral activity in the previous 3 months. The patients were randomly assigned in a double blind fashion either to $150 \mathrm{IU}$ oral $\mathrm{nHuIFN}-\alpha$ /day or placebo. The duration of treatment was extended from 28 weeks to 60 weeks 9 months after enrolment had started. At that time 112 subjects had already received 28 weeks of treatment and been discontinued from the study.

Results: Both study groups were comparable with respect to all baseline characteristics studied, except that the nHuIFN- $\alpha$ group had slightly lower absolute CD4+ lymphocyte counts (median $\left.60.7 \times 10^{6} / 1\right)$ than the placebo group (median $\left.85.3 \times 10^{6} / 1\right)(\mathrm{p}=0.033)$. Therefore, all analyses were adjusted for CD4+ lymphocyte counts at entry. In both treatment groups there was relentless progression of HIV disease. Subjects treated with nHuIFN- $\alpha$ and placebo had similar mortality, disease progression rates, decline of CD4+ lymphocyte counts and Karnofsky performance scores, and prevalence of symptoms. No patient reverted to HIV-l seronegative antibody status. Serious adverse events were not seen. Quality control of the study medication documented that the active drug indeed contained IFN- $\alpha$ activity.

Conclusions: The current large, randomised, double blind, placebo controlled study did not show any benefit from oral treatment with $150 \mathrm{IU}$ nHuIFN- $\alpha /$ day in a population of African patients with symptomatic HIV infection.

(Sex Transm Inf 1998;74:265-270)

Keywords: HIV infection; AIDS; interferon alfa

\section{Introduction}

Interferon alfa (IFN- $\alpha$ ) exhibits dose related in vitro activity against human immunodeficiency virus (HIV), with complete inhibition of HIV replication at IFN- $\alpha$ concentrations $\geqslant 256$ $\mathrm{IU} / \mathrm{ml}$ even in chronically infected cell lines. ${ }^{1-6}$ In vitro the $90 \%$ inhibitory concentrations with concomitant administration of zidovudine and IFN- $\alpha$ were $0.02 \mu \mathrm{mol}$ and $32 \mu \mathrm{IU} / \mathrm{ml}$, respectively.

Treatment of patients with AIDS related Kaposi's sarcoma with high dose $\left(27-36 \times 10^{6}\right.$ IU/day) subcutaneously administered IFN- $\alpha$ has resulted in tumour responses, rises in peripheral blood CD4+ lymphocyte counts, and significant decreases in serum p24 antigen levels, particularly in those patients with initial CD4+ cell counts above $50 \times 10^{6} / 1 .^{89} \mathrm{~A}$ randomised, double blind, placebo controlled trial, comparing placebo, $3 \times 10^{6} \mathrm{IU}$ and $36 \times 10^{6}$ IU of IFN- $\alpha$ intramuscularly or subcutaneously three times a week, in a population of AIDS patients without Kaposi's sarcoma and a median CD4+ cell count of approximately $50 \times$
$10^{6} / 1$, showed no clear benefits for either dose of IFN- $\alpha$ therapy. ${ }^{10}$ In a randomised, double blind, placebo controlled study, in patients with asymptomatic HIV infection who had CD4+ cell counts of $\geqslant 400 \times 10^{6} / 1$, IFN- $\alpha 35 \times 10^{6}$ IU/day subcutaneously, showed clear antiretroviral effects. ${ }^{11}$ In asymptomatic HIV infected subjects, decreases of serum p24 antigen levels have been attained with IFN- $\alpha$ dosages as low as $1.5 \times 10^{6} \mathrm{IU} /$ day, subcutaneously. ${ }^{12}$

In mid-1990, Kenyan investigators reported that oral treatment with an extremely low dose (150 IU/day) of nHuIFN- $\alpha$ (called Kemron by the investigators) of patients with AIDS or AIDS related complex (ARC), resulted within 10 weeks in complete alleviation of symptoms commonly associated with ARC and AIDS and resolution of opportunistic infections without additional treatment in all patients. Loss of HIV antibody seropositivity ("serodeconversion") was reported to occur in approximately $10 \%$ of the patients. ${ }^{13}$ Subsequent small studies failed to substantiate these spectacular claims. $^{14-16}$ 
In September 1990, the World Health Organisation (WHO) called a meeting of researchers, representatives of national drug regulatory agencies, and pharmaceutical manufacturers to examine the conflicting evidence on the efficacy of low dose oral IFN- $\alpha$. The data presented at this meeting were not conclusive and the expert committee recommended that WHO should commission a randomised double blind placebo controlled study of sufficient sample size to establish whether low dose oral IFN- $\alpha$ was efficacious in the treatment of symptomatic HIV infection. ${ }^{17}$ In this paper we report the findings of this study.

\section{Methods}

PATIENT CHARACTERISTICS

The study was conducted in the Department of Medicine at Makerere University Medical School, Mulago Hospital in Kampala, Uganda. The majority of patients recruited into the study came from either the AIDS clinic of Mulago hospital or The AIDS Support Organisation (TASO). Drugs on the WHO list of essential drugs were provided for the management of infections occurring during the trial period.

Men and non-pregnant women at least 15 years of age were eligible to enter the study if they met the following criteria: had HIV-1 infection documented by enzyme linked immunosorbent assay (ELISA) and confirmed by a western blot; were in stage $2-4$ of the WHO clinical staging system for HIV infection ${ }^{18}$; had an absolute peripheral blood CD4+ lymphocyte count of less than $300 \times 10^{6} / 1$ on two occasions more than 1 week apart; had a Karnofsky performance score of 50 or more; had serum urea, creatinine, and aspartate aminotransaminase (ASAT) levels of less than five times the upper limit of normal; gave written informed consent to participate in the study; and lived within 10 miles of the study site. Subjects were excluded if they were taking or had been taking any drugs with known antiretroviral activity in the previous 3 months.

\section{STUDY DESIGN}

This was a randomised, double blind, placebo controlled study. Subjects were given a unique incremental study number at enrolment, corresponding to their individual prepacked and prelabelled supply of study drugs, which could either be active drug or placebo, according to the randomisation code generated by the WHO. Each treatment arm initially consisted of 200 subjects, which was later revised to 280 on the recommendation of the Data and Safety Monitoring Board (DSMB) following a preplanned interim analysis in February 1992.

TREATMENT REGIMEN

The study drugs were tablets containing either 150 IU nHuIFN- $\alpha$ (Hayashibara Biochemical Laboratories, Okayama, Japan) stabilised in an anhydrous polysaccharide carrier, or matching placebo, to be taken daily for 28 weeks (extended to 60 weeks after a protocol amendment in February 1992). Patients were in- structed to place the tablet in the mouth and to allow it to dissolve in the saliva for 2-3 minutes, massaging gently with the tongue to facilitate dissolution and effective mucosal contact. They were encouraged to retain the saliva in the mouth for at least 30 minutes before swallowing it.

\section{CRITERIA FOR RESPONSE}

Primary measures of outcome were death, deterioration of health severe enough to warrant withdrawal from the study, and time to more advanced WHO clinical stage. Secondary measures of outcome were changes in body weight, subjective symptoms, Karnofsky performance scores, CD4+ lymphocyte counts, and adverse events. Loss of anti-HIV seropositivity was assessed at week 12 .

\section{SUBJECT TREATMENT AND EVALUATION}

The pretreatment evaluation included a medical history, a physical examination, assessment of HIV serostatus, peripheral blood CD4+ lymphocyte counts, a full blood count (FBC, using a Coulter counter model T540), and serum urea, creatinine, and ASAT levels by direct analysis methods.

The baseline CD4+ cell count was taken to be the average of three CD4+ lymphocyte count readings, which were obtained 2 weeks and 1 week before enrolment and at study entry. CD4+ lymphocyte counts were done using an adaptation of the immunoalkaline phosphatase method for blood smear. ${ }^{19}$ The slides were prepared and stained within 1 hour of collecting the blood and stored at $-20^{\circ} \mathrm{C}$ for reading at a later time.

HIV serology was done using a competitive enzyme assay (Wellcozyme HIV-1, Wellcome Diagnostics, Dartford, Kent) and confirmed by a western blot (Diagnostic Biotechnology HIV-1 BLOT, Singapore) according to the WHO guidelines. ${ }^{20}$

After entry into the study, the subjects were seen every 2 weeks for 28 weeks. After the interim analysis in February 1992, the protocol was amended such that treatment was extended to 60 weeks. Patients were seen every 4 weeks from weeks 28 to 60 .

On each visit symptoms and signs of HIV disease progression, incident HIV or AIDS related conditions, and incident adverse events were recorded. FBC and CD4+ lymphocyte counts were done every 2 weeks up to 12 weeks, at 18 and 24 weeks, and every 4 weeks up to 60 weeks. HIV serology was repeated after 12 weeks of treatment. Patients who failed to attend scheduled follow up visits were visited at home by the counsellor or one of the study doctors to ascertain the reason for defaulting, for on site clinical assessment, and to facilitate attendance at the study clinic.

\section{OUALITY CONTROL OF STUDY MEDICATION}

The IFN- $\alpha$ activity of the study medication was assessed at production, and blind tested twice during and at the end of the trial on a random sample of vials, by Hayashibara Biochemical Laboratories, at Fujisaki Institute, Okayama, Japan, and by the National Cancer Institute, 
Table 1 Baseline characteristics on admission

\begin{tabular}{lll}
\hline Variable & $n$ HuIFN- $a(n=279)$ & Placebo $(n=280)$ \\
\hline Mean age (range) & $31.9(17-67)$ & $31.218-59)$ \\
Sex: male (\%) & $129(46.2)$ & $123(43.9)$ \\
Cough (\%) & $132(47.3)$ & $156(55.7)$ \\
Itching (\%) & $188(67.4)$ & $181(64.6)$ \\
Blurred vision (\%) & $33(11.8)$ & $38(13.6)$ \\
Gastrointestinal symptoms (\%) & $165(59.1)$ & $149(53.2)$ \\
Joint or muscle pain (\%) & $124(44.4)$ & $127(45.4)$ \\
General symptoms (\%) & $199(71.3)$ & $199(71.1)$ \\
Mean body weight (range) & $51.8(32.0-85.5)$ & $51.7(31.0-85.0)$ \\
Mean Karnofsy score (range) & $82.666(50-90)$ & $82.8(20-90)$ \\
Mean haemoglobin (g/l) (range) & $10.9(5.4-18.0)$ & $11.0(6.1-17.5)$ \\
Median absolute CD4+ lymphocyte count (range) & $60.7(0.0-577)^{\star}$ & $85.3(0.0-562)^{\star}$ \\
Median percentage CD4+ lymphocytes (range) & $3.9(0.0-27.7)^{\star \star}$ & $4.8(0.0-27.2)^{\star \star}$ \\
WHO clinical stage & & \\
$\quad$ Stage 2 (\%) & $29(10.4)$ & $25(8.9)$ \\
Stage 3 (\%) & $112(40.1)$ & $124(44.3)$ \\
Stage 4 (\%) & $138(49.5)$ & $131(46.8)$ \\
\hline
\end{tabular}

General symptoms = tiredness, fever, or night sweats.

Gastrointestinal symptoms = loss of appetite, pain on swallowing, nausea, vomiting, or diarrhoea ${ }^{\star} \mathrm{p}=0.033 ;{ }^{\star \star} \mathrm{p}=0.065$.

Frederick Cancer Research and Development Centre, Frederick, Maryland, USA. Ten lozenges were sampled from each vial and assayed in duplicate, as described previously. ${ }^{21}$

STATISTICAL ANALYSIS

The two groups were compared on admission using the $\chi^{2}$ test, the $t$ test, or the MannWhitney $U$ test. Life table methods were used to analyse survival rates and disease progression. Survival rates were adjusted for a difference in baseline CD4+ lymphocyte counts using proportional hazards regression. Logistic regression was used to analyse symptoms on follow up, while repeated measures analysis of variance was used to analyse log transformed CD4+ lymphocyte counts, body weight, and Karnofsky scores.

Patients who discontinued from the study were retained in the analyses of CD4+ lymphocyte counts, body weight, and Karnof-

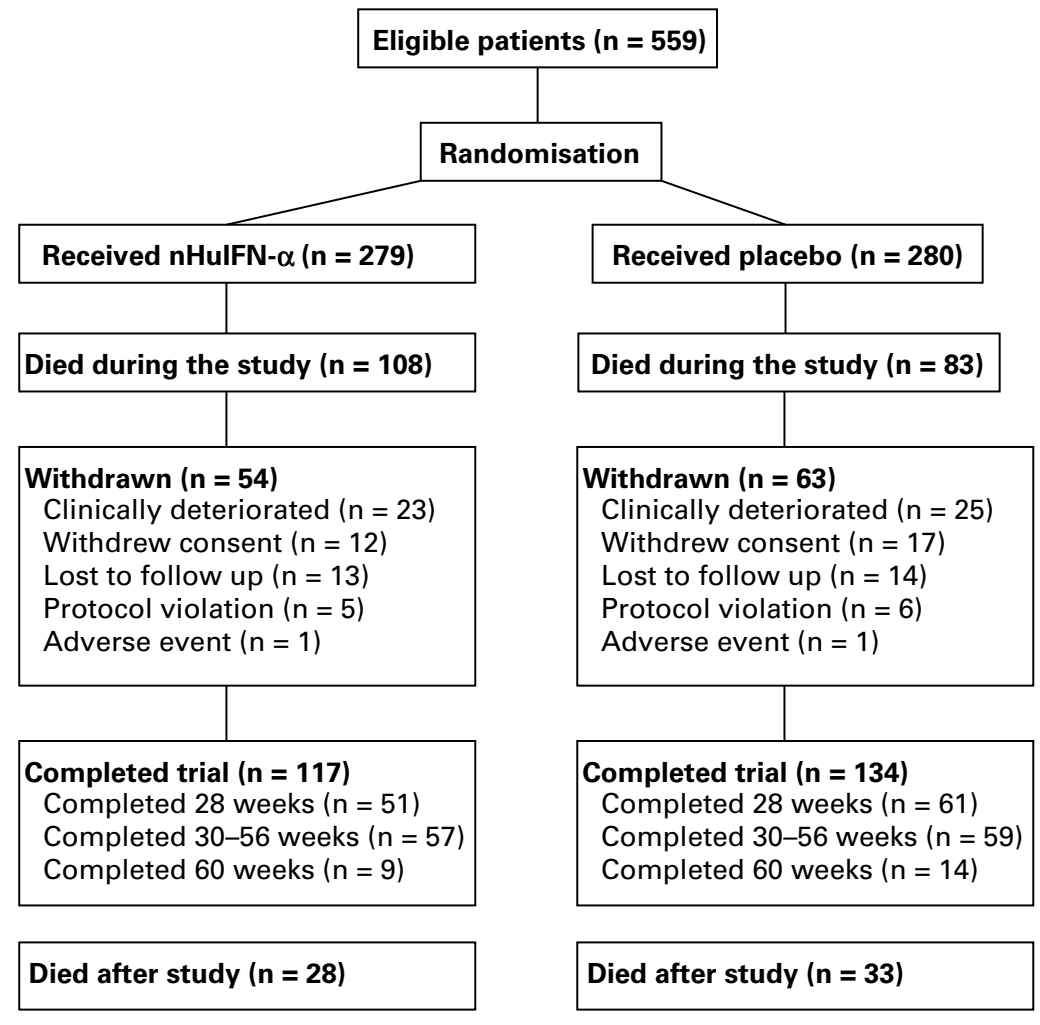

Figure 1 Flow chart describing progress of patients through the trial. sky scores after discontinuation. Their CD4+ lymphocyte counts and body weight were assumed to remain the same as at their last follow up visit; Karnofsky scores were treated in the same way unless the patient died, when they were set to 0 .

\section{Results}

STUDY POPULATION

Between 10 June 1991 and 6 July 1992, 560 subjects were enrolled in the study. One subject was found after admission to have been HIV 1 seronegative at entry and was excluded from the data analysis. Two hundred and seventy nine subjects received nHuIFN- $\alpha$ and 280 placebo.

Table 1 shows the baseline characteristics of the population at study entry. There were no significant differences between the two groups at the time of entry with respect to age, sex, WHO clinical stage, body weight, Karnofsky performance score, symptoms, haemoglobin, or biochemistry results (not shown). Although the WHO clinical stage at admission did not differ significantly between the two groups, nine subjects in the nHuIFN- $\alpha$ group had Kaposi's sarcoma at entry, compared with two in the placebo group $(\mathrm{p}<0.05)$.

The $n H u I F N-\alpha$ group had lower absolute CD4+ lymphocyte counts (median $60.7 \times$ $10^{6} / 1$ ) than the placebo group (median $85.3 \times$ $\left.10^{6} / 1\right)(\mathrm{p}=0.033)$. However, the CD4+ percentage values did not differ between groups at the $5 \%$ level of significance $(p=0.065)$.

\section{FOLLOW UP AND STUDY DISCONTINUATION}

The original treatment period was 28 weeks. A preplanned interim analysis, conducted in February 1992, showed a divergence between the two groups' survival rates which, however, was not statistically significant. The DSMB therefore recommended extending treatment to 60 weeks. By this time, 112 subjects had already completed 28 weeks' treatment and been discontinued from the trial ( 51 from the nHuIFN- $\alpha$ group, and 61 from the placebo group). The study was closed on 15 January 1993, 30 weeks after enrolment of the last subject. At the end of the study 23 subjects (nine in the nHuIFN- $\alpha$ group and 14 in the placebo group) had completed the 60 weeks of treatment, while 116 (57 in the nHuIFN- $\alpha$ and 59 in the placebo group) had completed between 30 and 60 weeks. The mean and median lengths of treatment for the $n \mathrm{HuIFN}-\alpha$ group were 6.0 and 6.4 months, while the placebo group they were 6.3 and 6.5 months, respectively.

One hundred and seventeen subjects left the study for reasons other than death. The reasons for discontinuation are summarised in figure 1 , and were similarly distributed in both treatment groups. Forty eight subjects discontinued the study because their condition deteriorated. They either became too sick to take the study medication or were transferred to their villages where it was not possible to continue supplying them with the study drug. Twenty nine subjects withdrew their consent, including 13 who felt 


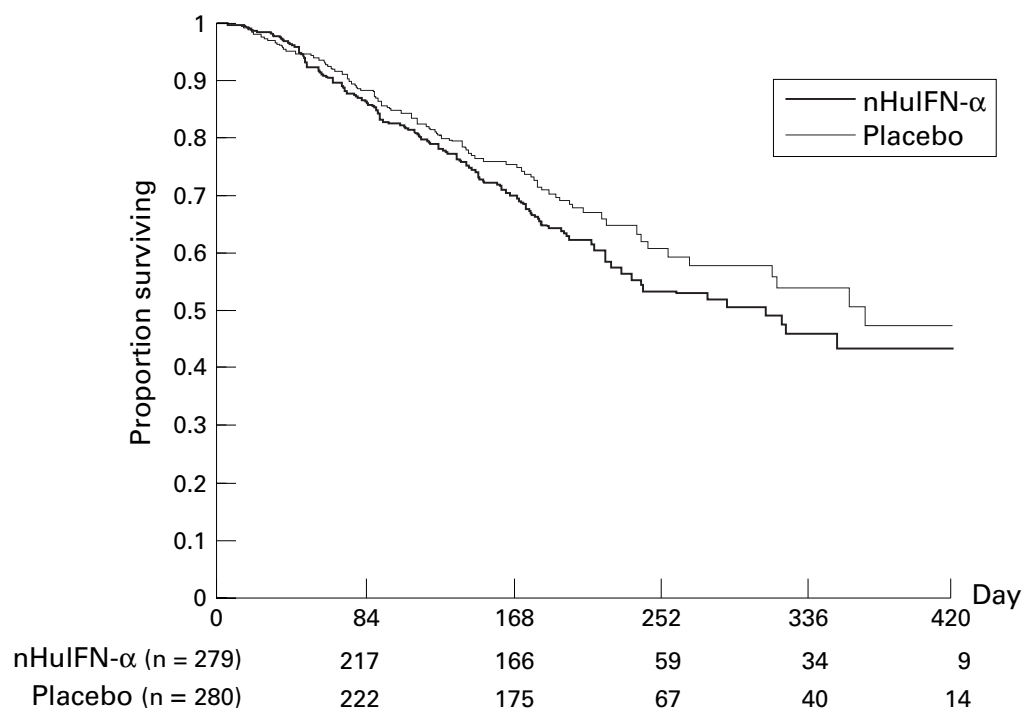

Figure 2 Survival by treatment group (adjusted for CD4+ lymphocyte count at entry).

they were not getting the improvement they had expected and one subject who withdrew from the study because she became pregnant.

Twenty seven subjects were lost to follow up and could not be traced. Eleven people violated the protocol because they missed two consecutive visits. Two subjects discontinued the study owing to symptoms which were perceived as adverse effects related to the study drug.

EFFICACY ASSESSMENT

Death

A total of 191 patients, 108 in the nHuIFN- $\alpha$ and 83 in the placebo group, died either during the period when they were on treatment or within 30 days of terminating the study. The unadjusted survival rate among subjects given nHuIFN- $\alpha$ was significantly lower than among placebo treated subjects $(\mathrm{p}=0.035)$; after adjustment for the difference in CD4+ lymphocyte counts at entry, however, the survival rates in the two groups were similar $(\mathrm{p}>0.3)$ (fig 2).

When the study was designed, there was no intention to record information about deaths which occurred more than 30 days after stopping treatment for other reasons. However, after the study the patients remaining in Kampala were followed up by one of the investigators (ETK in his routine HIV clinic, and additional information on their vital status was available from this source). In addition to the 191 patients who died while on study treatment or within 30 days of stopping treatment, the investigator was aware of another 61 deaths (28 in the nHuIFN- $\alpha$ group, and 33 in the placebo group) more than 30 days after treatment was stopped. In the nHuIFN- $\alpha$ group, 11 of these patients had discontinued from the study because their condition deteriorated, two had withdrawn consent, and 15 had completed 28 weeks of treatment in the study's first phase. In the placebo group, seven patients had discontinued because their condition deteriorated, four had withdrawn consent, four were discontinued because of a protocol violation, one had discontinued because of an adverse event, and 17 had completed 28 weeks of treatment in the study's first phase. When these deaths were included in analysis, there were no significant differences in the survival rates between the two groups, either with or without adjustment for the difference in CD4+ lymphocyte counts at entry.

It was not possible to establish accurately all the causes of death because of insufficient diagnostic facilities. Nevertheless, categories of causes of death (gastrointestinal, respiratory and central nervous system diseases, Kaposi's sarcoma, others, and unknown) were similarly distributed in both treatment groups. After the interim analysis in February 1992, the DSMB recommended that deaths should be recorded as HIV related or not. This information was collected for the last 99 deaths. Only one death in the placebo group, was not clearly HIV related: the patient committed suicide.

\section{Disease progression}

Rates of progression to a more advanced WHO clinical stage, clinical deterioration, or death did not differ between study groups, either before or after adjustment for the difference in CD4+ lymphocyte count at entry (both $p$ $>0.3$ ) (fig 3A).

Disease progression rates also did not differ between groups when patients were stratified by WHO clinical stage at enrolment (all $\mathrm{p}$ $>0.2$ ) (figs $3 \mathrm{~B}, \mathrm{C}, \mathrm{D}$ ).

\section{Change in symptoms}

There were no differences between the two treatment groups at any time with respect to the prevalence of symptoms. At least $95 \%$ of the nHuIFN- $\alpha$ group and $93 \%$ of the placebo group patients were symptomatic at each follow up visit. The prevalence of general symptoms (tiredness, fever, and night sweats) varied between $68 \%$ and $78 \%$ in the nHuIFN- $\alpha$ group and between $65 \%$ to $71 \%$ in the placebo group. The prevalence of gastrointestinal symptoms (loss of appetite, pain on swallowing, nausea, vomiting, and diarrhoea) varied between $59 \%$ and $70 \%$ in the nHuIFN- $\alpha$ group and between $53 \%$ and $64 \%$ in the placebo group.

\section{Changes in CD4+ lymphocyte counts}

The difference in CD4+ lymphocyte counts, observed at baseline, persisted throughout the study. During treatment there was a similar steady decline in the CD4+ lymphocyte cell counts in the two groups (table 2). When the CD4+ lymphocyte counts during treatment were adjusted for the count at baseline, there were no significant differences in the CD4+ lymphocyte counts between study groups.

\section{Changes in Karnofsky performance score and} body weight

Karnofsky performance scores decreased uniformly in both groups throughout the study period: at weeks $0,12,24,36,48$, and 60 the mean Karnofsky scores of subjects in the nHuIFN- $\alpha$ group were $83,71,58,50,48$, and 45 , respectively, and in the placebo group 83, $74,63,57,53$, and 52 respectively. 


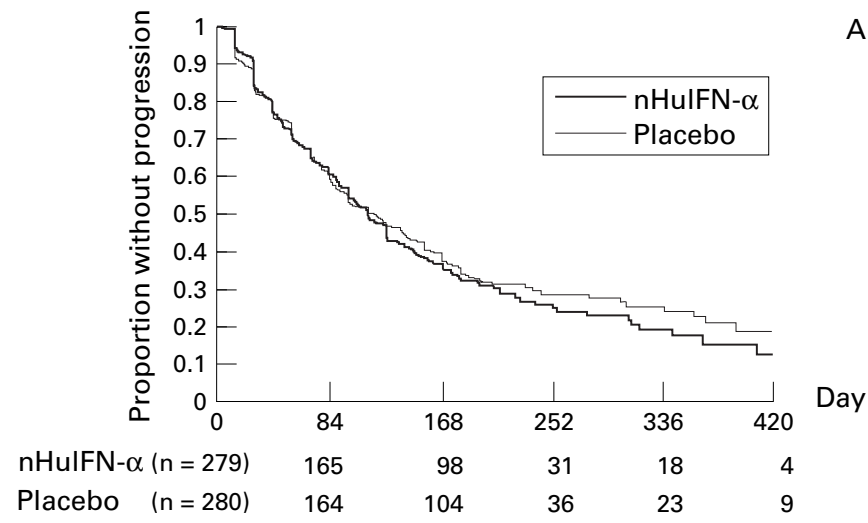

A
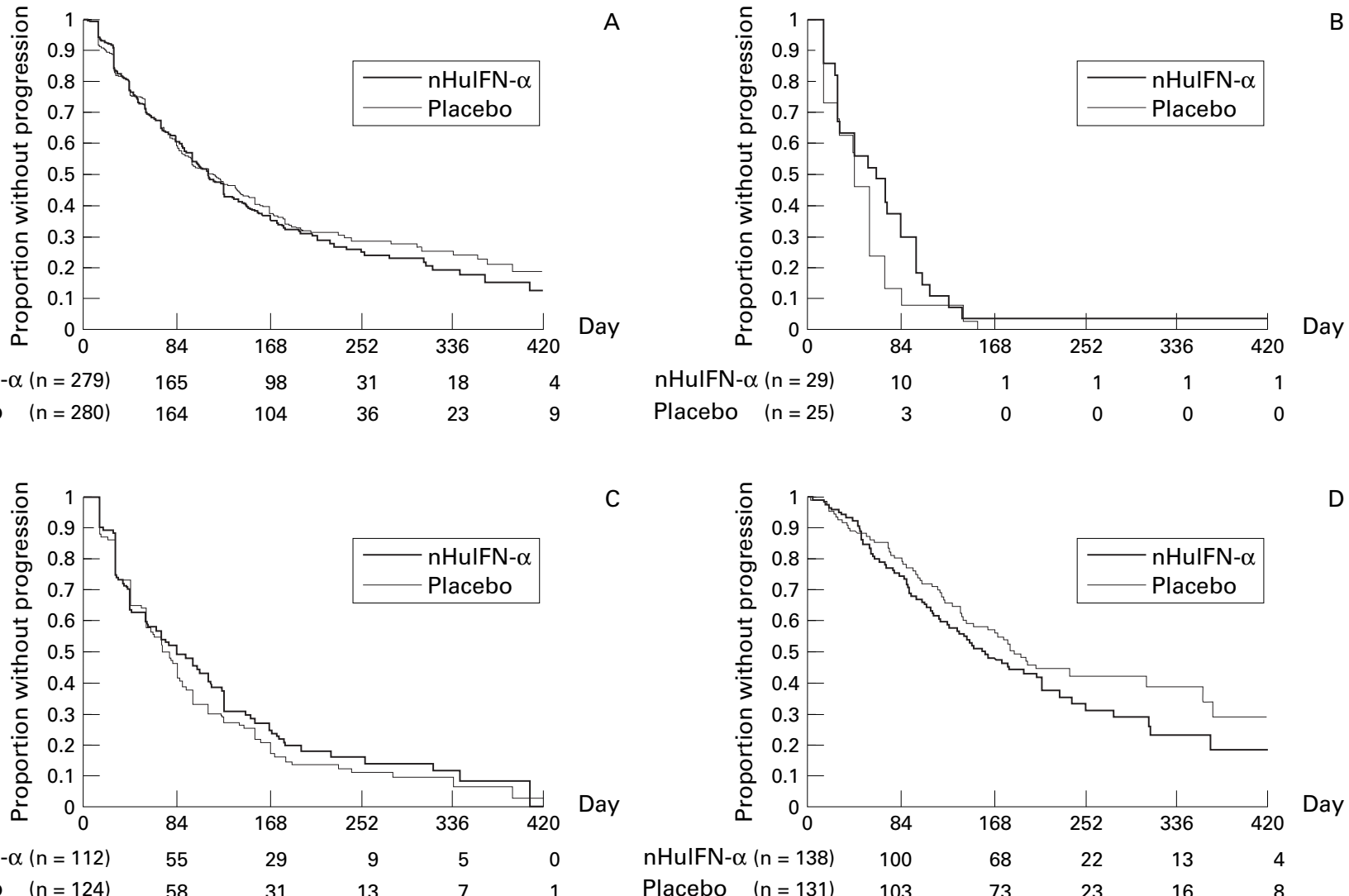

Placebo $\quad(n=124) \quad 58$

Placebo $\quad(\mathrm{n}=131) \quad 103$

Figure 3 Disease progression (more advanced stage, deterioration, or death) by treatment group (adjusted for CD4+ lymphocyte count at entry). (A) All randomised patients. (B) Patients with WHO stage 2 disease on admission. (C) Patients with WHO stage 3 HIV disease on admission. (D) Patients with WHO stage $4 \mathrm{HIV}$ disease on admission.

There was no significant change in body weight in the two study groups. Mean body weight varied from 52 to $55 \mathrm{~kg}$ in the nHuIFN- $\alpha$ group, and from 52 to $58 \mathrm{~kg}$ in the placebo group.

HIV serodeconversion

No patient reverted to HIV-1 seronegative antibody status in the two groups.

ADVERSE EVENTS

One subject in the nHuIFN- $\alpha$ group developed symptoms of chest pain, dizziness, and abdominal pain from the day she started taking the study medication. These symptoms continued for 3 days until she stopped the medication. She reported similar symptoms on restarting the medication 2 days later and was advised to stop the treatment. One subject in the placebo group had been taking the drug for 5 weeks when she developed diarrhoea which she associated with the study drug. The diarrhoea stopped when she stopped the medication a week later. There were no other discontinuations from the study as a result of adverse events.

Table 2 Median absolute CD+ lymphocyte counts during follow up (range)

\begin{tabular}{lll}
\hline Week & $n$ HulFN-a & Placebo \\
\hline Baseline & $60.7(0-577)$ & $85.3(0-562)$ \\
12 & $26.0(0-960)$ & $42.0(0-748)$ \\
24 & $20.0(0-655)$ & $46.5(0-966)$ \\
36 & $18.0(0-725)$ & $37.0(0-1014)$ \\
48 & $15.0(0-670)$ & $32.0(0-1144)$ \\
60 & $15.0(0-670)$ & $32.0(0-782)$ \\
\hline
\end{tabular}

QUALITY CONTROL OF STUDY MEDICATION The IFN- $\alpha$ activity of verum and placebo tablets was assessed on 10 vials of each at production, on six verum and three placebo vials in October 1991, on three vials of each in April 1992, and on six verum and five placebo vials in September 1992. The mean IFN- $\alpha$ activity of verum tablets was 160 (SD 8) IU, 154 (12) IU, 145 (6) IU, and 135 (11) IU, respectively. None of the placebo tablets exhibited IFN- $\alpha$ activity. Independent analysis in a laboratory in the United States confirmed that verum tablets had IFN- $\alpha$ activity.

\section{Discussion}

The current large, randomised, double blind, placebo controlled study did not show any benefit from oral treatment with low dose $\mathrm{nHuIFN}-\alpha$ in a population of African patients with symptomatic HIV infection. Except for a small difference in survival, which was slightly worse in the nHuIFN- $\alpha$ group, there were no differences in disease progression, symptomatology, or any of the other efficacy parameters between treatment groups. CD4+ lymphocyte counts declined relentlessly in both groups. When the analyses of survival and disease progression were adjusted for CD4+ lymphocyte counts at entry, the difference in survival between both study groups was no longer significant and no new significant differences emerged. No loss of HIV antibody seropositivity was observed.

It may be argued that the patients in this study were too severely immunocompromised to benefit from IFN- $\alpha$ therapy. ${ }^{8-11}$ 
Nevertheless, the results are in striking contrast with those from Kenya ${ }^{13}$ in a population with similarly advanced HIV infection in which an identical formulation, dose, and route of drug administration of IFN- $\alpha$ was used. Moreover, the present study, patients who at entry had only minor symptoms of HIV infection (WHO clinical stage 2), also showed similar disease progression rates in both treatment groups.

Interferons are generally not believed to be orally bioavailable, and are rapidly denatured upon contact with gastric secretions since they are proteins. $^{22}$ To avoid gastric denaturing, patients were instructed to keep the tablets in the mouth as long as possible in order to achieve maximum absorption within the mouth through mucosal contact. However, no known oral IFN- $\alpha$ receptor has been isolated to date and therefore no one knows how much of the orally taken dose is actually absorbed..$^{22}$ Even if there were substantial oral absorption of $n H u I F N-\alpha$, it is hard to imagine that a daily dose of 150 IU would exert any noticeable effect on HIV replication. In vitro IFN- $\alpha$ concentrations of $\geqslant 4 \mathrm{IU} / \mathrm{ml}$ are required to achieve some inhibition of HIV replication, ${ }^{1}$ and even when a synergistic combination of zidovudine and IFN- $\alpha$ is used, the $90 \%$ inhibitory concentration for IFN- $\alpha$ is $32 \mathrm{IU} / \mathrm{ml}^{7}$ Thus it is also highly unlikely that patients at earlier stages of HIV infection will benefit from treatment with low dose oral IFN- $\alpha$. Further efforts to evaluate the effect of low dose oral IFN- $\alpha$ therapy in patients with HIV infection would be better redirected to more rational approaches to the treatment of this deadly infection.

Funding: This study was funded by the Global Programme on AIDS of the World Health Organisation.

The authors wish to thank Hayashibara Biochemical Laboratories, Okayama, Japan, for providing the study drugs free of tories, Okayama, Japan, for providing the study drugs free of
charge and carrying out medication quality control studies: The National Cancer Institute, Frederick Cancer Research and National Cancer Institute, Frederick Cancer Research and
Development Centre, Frederick, Maryland, USA, for carrying out independent medication quality control studies: the man people who contributed to the success of this study, with special mention of doctors Michael Sempa and John Nkusi, staff nurses Norah Kagusa, Sarah Ndagire, and Proscovia Balya, counsellor Godfrey Sempa, laboratory technicians Herbert Mwesigye, Sam Nsobya, Fred Kabikira, and Olive Nakakeeto, data clerks Stephen Katabalwa and the late Stella Mukundane, and cleaner Betty Nansubuga, all in Uganda; and Ronan Doorly, Seng Pheth Kristoffersson, at WHO, who helped with data entry and presentation graphics.

Contributors: ET Katabira was the principal investigator of the study and in charge of writing the manuscript; NK Sestudy and in charge of writing the manuscript; NK Se-
wankambo was the co-principal investigator and wrote some of the sections of the manuscript; RD Mugerwa was the co-principal investigator and wrote some of the sections of the manuscript; EM Belsey was the statistician who analysed the data, wrote and revised the results section; FX Mubiru was the medical officer who was heavily involved in the patient review and follow up as well as data collection; C Othieno was the medical officer who was in charge of the study counsellors and ensuring that all patients in the study were traced; P Kataah was in charge of the laboratory aspect of the study and was involved in the development of the study. He was also involved in the writing of the manuscript. $M$ Karam was in charge of the development of the study at the WHO and argited the manuscript; M Youle was the first WHO consultant who was responsible for setting up the study in Uganda and was at the study site for over $50 \%$ of the study period; JH Perriens took over from Dr M Youle and completed the study. He was responsible for the data analysis in Geneva and participated fully in the writing of the manuscript; JMA Lange was responsible overall for the study for GPA and was also involved in writing the manuscript.

1 Ho DD, Hartshorn KL, Rota TR, et al. Recombinan human interferon alpha-A suppresses HTLV-III replication in vitro. Lancet 1985; i:602-4.

2 Yamamoto JK, Barré-Sinoussi F, Bolton V, et al. Human alpha and beta interferon but not gamma suppress the in vitro replication of LAV, HTLV-III, and ARV-2. F Interferon Res 1986;6:143-52.

3 Hartshorn KL, Neumeyer D, Vogt MW, et al. Activity of interferons alpha, beta, and gamma against human immunodeficiency virus replication in vitro. AIDS Res Hum nodeficiency virus replication

4 Yamada O, Hattori N, Kurimura $\mathrm{T}$, et al. Inhibition of growth of HIV by human natural interferon in vitro. AIDS Res Hum Retroviruses 1988;4:287-94

5 Poli G, Orenstein JM, Kinter A, et al. Interferon-alpha but not AZT suppresses HIV expression in chronically infected cell lines. Science 1989;244:575-7.

6 Kornbluth RS, Oh PS, Munis JR, et al. Interferons and bacterial lipopolysaccharide protect macrophages from productive infection by human immunodeficiency virus in vitro. f Exp Med 1989;169:1137-51.

7 Hartshorn KL, Vogt MW, Ting-Chao C, et al. Synergistic inhibition immunodeficiency virus in vitro by azidothymidine and recombinant alpha interferon. Antimicrob Agents dine and recombinant alpha in

8 De Wit R, Eeftinck-Schattenkerk JKM, Boucher CAB, et al. Clinical and virological effects of high-dose recombinant interferon-alpha in disseminated AIDS-related Kaposi's sarcoma. Lancet 1988;ii:1214-7.

9 Lane HC, Kovacs JA, Feinberg J, et al. Anti-retroviral effects of interferon-alpha in AIDS-associated Kaposi sarcoma Lancet 1988;ii:1218-22.

10 Interferon Alpha Study Group. A randomised placebocontrolled trial of recombinant human interferon alpha $2 \mathrm{a}$ in patients with AIDS. 7 Acquir Immunodefic Syndr 1988;1: $111-8$.

11 Lane HC, Davey V, Kovacs JA, et al. Interferon-alpha in patients with asymptomatic human immunodeficiency patients with asymptomatic human immunodeficiency virus (HIV) infection: a randomised

12 Orholm M, Pedersen C, Mathiesen L, et al. Suppression of p24 antigen in sera from HIV-infected individuals with low-dose alpha-interferon and zidovudine: a pilot study. AIDS 1989;3:97-100.

13 Koech DK, Obel AO. Efficacy of KEMRON (low dose ora natural human interferon alpha) in the management of HIV-1 infection and acquired immunodeficiency syndrome (AIDS). E Afr Med f 1990;67:SS64-70.

14 Kaiser G, Jaeger H, Birkmann J, et al. Low-dose oral natural human interferon- $\alpha$ in 29 patients with HIV-1 infection: a double-blind, randomised, placebo-controlled trial. AIDS 1992;6:563-9.

15 Hulton MR, Levin DL, Freedman LS. Randomised, placebo-controlled. double-blind study of low-dose oral placebo-controlled. double-blind study of low-dose oral interferon- $\alpha$ in HIV-1 antibody positive
Immune Def Syndr 1992;5:1084-90.

16 Sperber SJ, Gocke DJ, Haberzettl CA, et al. Low-dose oral recombinant interferon- $\alpha \mathrm{A}$ in patients with HIV-1 infection: a blinded pilot study. AIDS 1993;7:693-7.

17 World Health Organisation. Low-dose oral interferon alfa in the management of AIDS: still an experimental drug. Press release WHO/47. Geneva: WHO, 14 September 1990.

18 World Health Organisation. Interim proposal for a WHO staging system for HIV infection and disease. Wkly Epidemiol Rec 1900;65:221-4.

19 Lisse IM, Whittle H, Aaby P, et al. Labelling of T cell subsets under field conditions in tropical countries. Adaptation of the immunoalkaline phosphatase staining method for of the immunoalkaline phosphatase staining method
blood smears. F Immunol Methods 1990;129:49-53.

20 World Health Organisation. Proposed WHO criteria for interpreting results from Western blot assays for HIV-1, HIV-2. and HTLV-I/HTLV-II. Wkly Epidem Rec 1990;37 $281-3$.

21 Johnston MD, Finter NB, Young PA. Dye uptake method for assay of interferon activity. Methods Enzymol 1981;78: 394-9.

22 Interim report: low-dose oral interferon alpha as a therapy for human immunodeficiencv virus infection (HIV-1): completed and on-going clinical trials. National Institutes of Health. Washington. April 1992. Re-edited 6/1/92. 